\title{
Anthropometric measurements at birth as predictor of low birth weight
}

\author{
Negar Sajjadian ${ }^{1}$, Hamideh Shajari ${ }^{1}$, Farnoosh Rahimi ${ }^{1}$, Ramin Jahadi ${ }^{2}$, Michael G. Barakat ${ }^{3}$
}

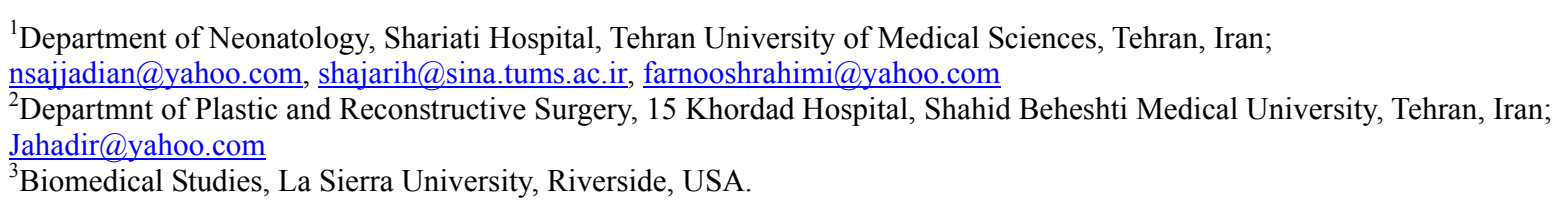

Received 22 May 2011; revised 16 July 2011; accepted 20 September 2011.

\begin{abstract}
In developing countries, low birth weight (BW < 2500 grams) accounts for $60 \%-80 \%$ of neonatal deaths. Early identification and referral of LBW babies for extra essential newborn care is vital in preventing neonatal deaths. Studies carried out in different populations have suggested that the use of newborn anthropometric surrogates of birth weight may be a simple and reliable method to identify LBW babies. previous studies reported correlation between birth weight to several anthropometric measurements and their predictive value. We aimed to evaluate the correlation between birth length, head, chest, and mid arm circumferences to birth weight. Methods: A cross sectional study has been conducted in SHARIATI Hospital in Tehran, from September 2008 to February 2009. All Consecutive full-term. Single ton, live born babies were included and anthropometric measurements carried out within $\mathbf{4 8}$ hours after birth by authors. Birth weight was measured by digital scale within the first 24 hours after delivery. Birth length by somatometer and head, chest, mid arm circumferences were measured 2 times by using plastic measuring tape. Result: Out of $\mathbf{5 0 0}$ newborn studied. $52.2 \%$ were male and $47.8 \%$ were female. The mean birth weight was 3195.4 \pm 399.9 gram and $3.8 \%$ of newborns were low birth weight. It was evident a positive correlation of birth weight to all such anthropometric measurements with the highest correlation coefficient for chest circumference ( $r$ : 0.74). By ROCAUC analyses, chest circumference (AUC $=0.91$, $95 \% \mathrm{Cl} 0.84$ to 0.97 ) and arm circumference ( $A U C=0.87,95 \% \mathrm{Cl} 0.79$ to 0.95 ) were identified
\end{abstract}

as the optimal surrogate indicators of LBW babies. The optimal cut-points for chest circumference and arm circumference to identify LBW newborns were $\geq 31.2 \mathrm{~cm}$ and $\geq 10.2 \mathrm{~cm}$ respectively. Conclusions: Chest and mid arm circumferences were the best anthropometric surrogates of LBW among studied Iranian population. Further studies are needed in the field to cross-validate our results. anthropometric values are simple, practicable, quick and reliable indicator for predicting LBW newborns in the community and can be easily measured by paramedical workers in developing nation.

Keywords: Low Birth Weight; Anthropometric Measurements; Neonatal Mortality

\section{INTRODUCTION}

Of the approximately four million global neonatal deaths that occur annually, 98\% occur in developing countries, where most newborns die at home while they are being cared by mothers, relatives, and traditional birth attendants [1]. About $38 \%$ of total under-five mortality occurs during the neonatal period and nearly three quarters of these deaths occur during the first week of life [2]. Globally, about one-sixth of all newborns are low birth weight (LBW, $<2500$ grams), which is single most important underlying risk factor for neonatal deaths $[1,3]$. Only about half of the newborns are weighed at birth and for a smaller proportion of them gestational age is known [4]. An estimated 18 million babies are born with LBW [5]. They account for $60 \%-80 \%$ of neonatal deaths [6]. Moreover, LBW babies who survive the critical neonatal period may suffer impaired physical and mental growth. Therefore, an early identification and prompt referral of LBW newborns is vital in preventing 
neonatal deaths. Available evidence from resource-poor settings shows that extra essential newborn care for LBW babies can reduce the number of neonatal deaths by $20 \%-40 \%$ [7]. Most neonatal deaths occur in the community and some interventions, including vitamin $\mathrm{A}$ supplementation, newborn skin cleansing with chlorhexidine and topical emollient therapy may be targeted preferentially to LBW infants to reduce mortality risk. Thus, continued efforts are required to describe optimal methods for identifying these high-risk infants in the community. In resource-poor settings, a large proportion of deliveries take place at home and birth-weight is most often not recorded. Therefore, there is a need to develop simple, inexpensive and practical methods to identify LBW newborns soon after birth [8]. One such method may be the use of anthropometric surrogates to identify LBW babies. A number of studies have focused on measuring the circumference of the head, chest, mid upper-arm, thigh or calf and observed the correlation with continuous measurements on a gold standard weighing scale (Bhargava et al., 1985; Singh et al., 1988; WHO Collaborative study of birth weight surrogates, 1993; Dhar et al., 2002). In general, chest circumference has performed better than other measures and has been recommended for continued investigation, although investigators have demonstrated correlations between birth weight and mid upper-arm circumference (Sauerborn et al., 1990, calf (Gupta et al., 1996) (Samal and Swain, 2001 or thigh (Sharma et al., 1989) that are as strong as with chest circumference.

Most suitable and reliable anthropometric surrogate to identify LBW Iranian newborns and its cut-off point to identify LBW newborns is not known. Therefore, we carried out this study with following objectives:

1) to identify a suitable anthropometric surrogate to identify LBW babies and

2) to determine its cut-off value to identify LBW babies.

\section{MATERIALS AND METHODS}

A cross sectional study was performed among all term single tone neonate born at SHARIATI hospital (Tehran, Iran), a reference center for high risk pregnancies, from September 2008 to February 2009.

The study group consisted of all consecutive full term-single tone newborn with gestational age of between 37 weeks and 41 weeks and 6 days as estimate by maternal last menstrual period (LMP) date and first trimester sonography when their differences are not more than week. Preterms were excluded because they are probably low birth weight.

These newborn were examined by the authors within their first 48 hours of life. Newborns whose mother pre- sented complications during pregnancy (preclampsia, Diabetes, addiction and smoking) or newborns with major congenital anomaly, hydropic feature or intrauterine growth retardation (IUGR) were excluded.

Birth weight with naked neonate in supine position was obtained soon after birth by digital scale with 10 gram subdivision. All other anthropometric Variable including chest, head, mid arm Circumferences were measured by non extendable measuring tape, with a width of $1.0 \mathrm{~cm}$ and subdivisions of $0.1 \mathrm{~cm}$. and birth length was measured by somatometer

Head circumference was obtained by placing tape along the largest occipitofrontal diameter along over the occiput and eyebrow.

The chest circumference was measured by placing measuring tape along the point of nipples. The mid arm circumference was obtained from the left arm with elbow at the mid point between acromion and olecranon, with the newborn was located in dorsal decubitus with arm lying laterally to the trunk.

The length was measured with the newborn in supine position with full extension of knee and distance between top of head and heel when pressed against a vertical surface and role on a stabilizing board was measured.

A total of two consecutive measurements were taken for each variable and the mean values were recorded.

Continuous variables are reported as mean and standard deviation while between-gender comparisons of continuous variables were performed using independent sample $t$-test. Pearson's product-moment correlation coefficient was used to assess the association between anthropometric measurements. Receiver operating characteristic (ROC) curves were used to evaluate the accuracy of different anthropometric measurements to predict LBW. Non parametric receiver operating characteristic analysis was done to compare the overall utility of anthropometric measurements for Identifying LBW infant. Multivariate linear regression with backward stepwise method was used for estimation of birth weight by anthropometric measurment.

Sensitivity, specificity were calculated at all cut-points for any anthropometric measurement. We choose as "optimum" the cut-point with the highest [(sensitivity + specificity)/2] ratio. This criterion was chosen to allow comparison with previous studies available in the literature. The data analysis was done by the spss version 11.5 and $A$ $P$-value $<0.05$ was considered statistically significant.

\section{RESULTS}

A total of 500 newborns (52.2\% male and $47.8 \%$ female) were studied.

The mean birth weight was $3195.48 \pm 399.92$ gram. $3.8 \%$ of patients were LBW. Summary measures of 
weight, and anthropometric variable are presented in Table 1. In our study there were significant differences in birth weight and anthropometric measurements between male and female newborns $(P$-value $<0.05)$ the males had higher birth weight and all anthropometric variable except mid arm to head circumference ratio.

The anthropometric measurements were correlated with birth weight with significant $p$ value, the maximum correlation with birth weight was observed for chest circumference $(\mathrm{r}=0.74)$ and the mid arm circumference $(\mathrm{r}$ $=0.70)$, head circumference $(\mathrm{r}=0.65)$. Length $(\mathrm{r}=0.61)$ and a weak correlation was seen with mid arm circumference to head circumference $(r=0.44)$ with the best correlation coefficient observed for the weight-chest circumference association.

Multivariate Linear regression analysis was conducted for predicting birth weight by anthropometric measurements (Table 2).

The best discrimination of LBW, as detected by ROCAUC, was obtained by chest circumference (AUC = $0.91,95 \%$ CI 0.84 to 0.97 ) followed by lenght (AUC = $0.89,95 \%$ CI 0.79 to 0.99 ), head circumference (AUC = $0.87,95 \%$ CI 0.80 to 0.94$)$, mid arm circumference (AUC $=0.87,95 \%$ CI 0.79 to 0.95 ) and mid arm to head circumference ratio (AUC $=0.74,95 \%$ CI 0.63 to 0.85 )

The sensitivity, specificity, for classifying infants into LBW status was shown in Table 3. An optimum cut off point identifying LBW were $31.2 \mathrm{~cm}$ for chest circumference $10.2 \mathrm{~cm}$ for mid arm circumference, $33.2 \mathrm{~cm}$ for head circumference, $48.5 \mathrm{~cm}$ for length and 0.3 for mid head circumference, $48.5 \mathrm{~cm}$ for length and 0.3 for mid arm to head circumference ratio (Table 3 ).

Table 1. Descriptive statistics of birth weight and anthropometric measurements.

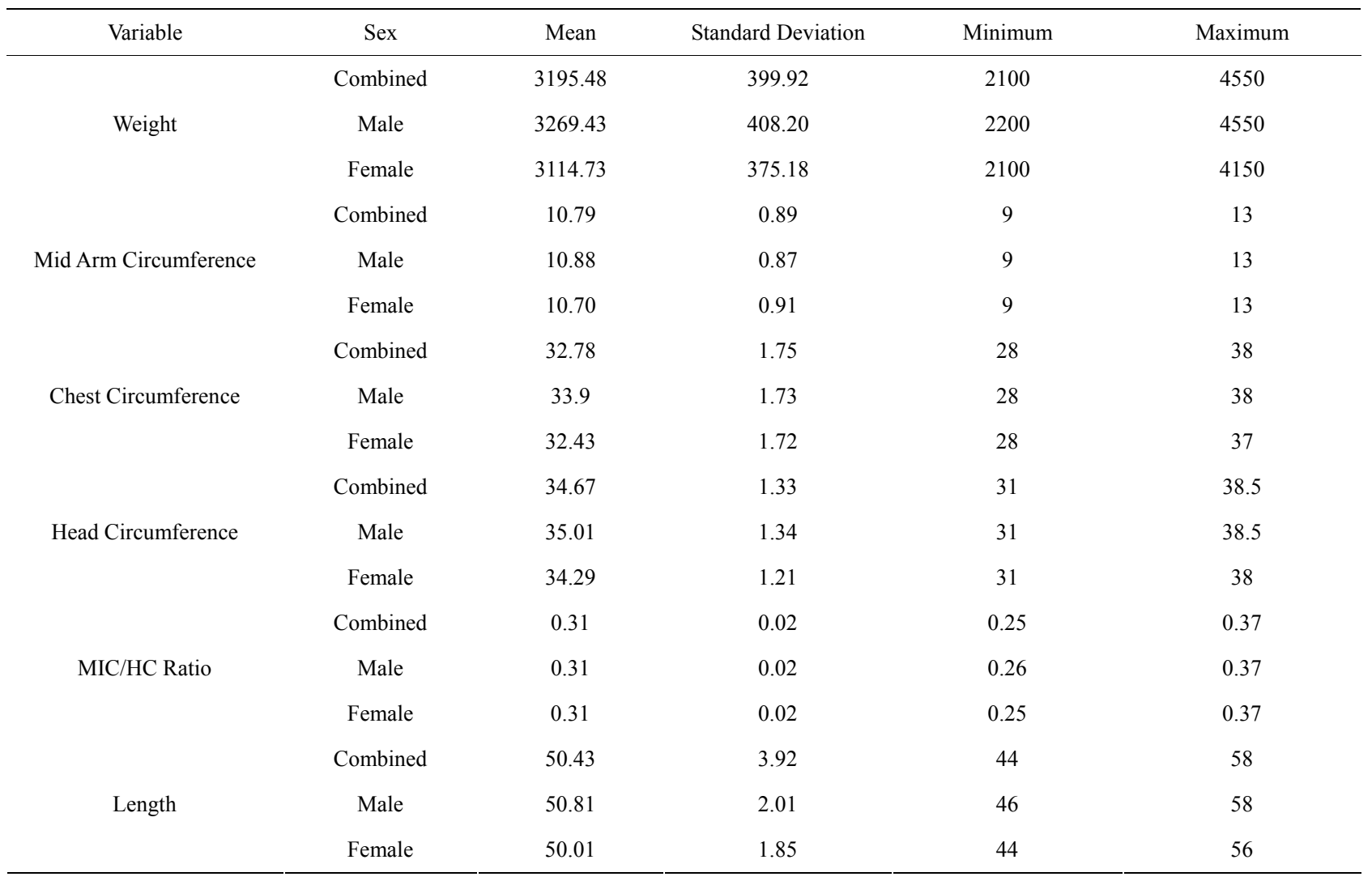

Table 2. Linear regression equation for estimation of birth weight.

\begin{tabular}{cccc}
\hline Variable & Regresion Equation & F (P-value) & Adjusted R $^{2}$ \\
\hline MAC & $\mathrm{BW}=-162.58+311.19(\mathrm{MAC})$ & $477.48(0.00)$ & 0.488 \\
$\mathrm{MAC} / \mathrm{HC}$ & $\mathrm{BW}=839.45+7569.4(\mathrm{MAC} / \mathrm{HC})$ & $121.210(0.00)$ & 0.194 \\
$\mathrm{CC}$ & $\mathrm{BW}=-2329.13+168.55(\mathrm{CC})$ & $606.38(0.00)$ & 0.548 \\
$\mathrm{HC}$ & $\mathrm{BW}=-3596.83+195.90(\mathrm{HC})$ & $371.23(0.00)$ & 0.426 \\
$\mathrm{LE}$ & $\mathrm{BW}=3041.77+123.67(\mathrm{~L})$ & $298.8(0.00)$ & 0.374 \\
\hline
\end{tabular}


Table 3. Sensitivity and specifity of optimum cutoff points of anthropometrics.

\begin{tabular}{cccc}
\hline Variable & Cut off limit & Sensitivity & Specifity \\
\hline Chest circumference & 31.25 & $84 \%$ & $81 \%$ \\
Head circumference & 33.25 & $73 \%$ & $85 \%$ \\
Mid arm circumference & 10.25 & $94 \%$ & $68 \%$ \\
Length & 48.5 & $84 \%$ & $88 \%$ \\
Mid arm circumference & 0.303 & $73 \%$ & $62 \%$ \\
\hline Head circumference & & & \\
\hline
\end{tabular}

\section{DISCUSSION}

The prime concern of the present study was to identify the best suitable surrogate parameter, proxy, to birth weight, which when used by the health personnel in domiciliary outreach will detect the maximum number of at risk infants for providing them with timely and needed intervention strategy.

The mean birth weight and anthropometrics in our population is higher than some previous mentioned study. A WHO multicenter study reported that the average birth weight was 2630, 2780 and 3840 for newborns in India, Nepal and Sri Lanka respectively [14]. Higher mean birth weight may be because only the full term singleton live births were included in our study. Previous studies did not specify such criteria $[11,16,19]$. Birth weights of the newborns born before completion of 37 weeks of gestation (full term) may also have been included in the studies cited above

Our data relieved that positive correlation between all studied parameters and birth weight is present. In our study there were significant differences in birth weight and anthropometric measurements between male and female newborns. Males had higher mean birth weight and anthropometrics than females. This finding is similar to Dhar study that strongest correlation was present between $\mathrm{CHC}$ and birth weight was observed $(\mathrm{r}=0.84)$. It may be due to large size of our population that make these difference significant however they lack clinical significance.

The percent of LBW in our population were lower than the Nepal $(8.5 \%)$ and Tanzania $(18 \%$ - $8 \%)$ study $[8,9]$. The reason of these finding may be related to different characteristics of population studied (genetic, nutritional, environmental background) and because we exclude all preterm neonates. In WHO collaborative study of birth weight surrogates Clear differences were seen between the centers in terms of the means and tenth centiles of both birth weight and the anthropometric measures. The values confirm the expected regional differences, since centers in South Asia, such as Delhi and Chandigarh, have on average the lowest values, whereas those in Europe, such as St. Peters. Many researchers have attempted to identify a suitable anthropometric surrogate to identify LBW babies which is reliable, simple, and logistically feasible in field conditions. Some studies have recommended that $\mathrm{CHC}$, MUAC and $\mathrm{HC}$ may be used as anthropometric surrogates to identify LBW babies [11-15,17-20]. Therefore we considered all these anthropometric measurement. In our study $\mathrm{CHC}$ was identified as a suitable surrogate to identify LBW babies.

In the present study, the maximum correlation with birth weight was observed for chest circumference $(r=$ $0.74)$ and the mid arm circumference $(r=0.70)$ so chest and mid-arm circumference had the best correlation with birth weight and are good predictors of LBW neonates. According to other studies (mullany et al., 2007 WHO Collaborative study of birth weight surrogates, 1993) The correlations between birth weight, arm circumference and chest circumference are high ranging from 0.60 to 0.95 and suggested that chest circumference is the Optimal anthropometric measure for establishing cutoffs for the identification of LBW infants $[9,19]$.

The preset study shows that chest circumference at a cut off limit of $31.2 \mathrm{~cm}$ is affective in detecting low birth weight infant with a sensitivity of $84 \%$ and specifity of $81 \%$. Cupta et al. showed cut off limit $30.1 \mathrm{~cm}$ with specifity 69 and sensitivity of $83 \%$ and Virdi et al. study cut off point of 30 with sensitivity 60 and specifity 0.3 $[12,13]$. A WHO collaborative study has recommended that $\mathrm{CHC}$ of 29 centimeters and 30 centimeters may identify "highly at risk" and "at risk" newborns respectively [19]. In our study maximum sensitivity and specificity for $\mathrm{CHC}$ was at $\mathrm{CHC}$ of 30.8 centimeters. The higher mean birth weight of newborns may be the reason for a slightly higher cut-off point obtained in our study. We considered only full-term deliveries, which was unlike earlier studies $[12,17,20]$.

Thus, it is evident from analysis of our data that chest circumference is the best suitable and simple surrogate parameter that could be used in the domiciliary outreach when it is impossible to record weight of baby at birth. For health personnel who are working in the community can use color coded tapes indicting weight $<2500$ grams.

We recommend the use of chest rather than arm circumference as a surrogate for birth weight for two reasons. First, it is simpler to measure identification of the nipple line is easier, making measurement more operationally feasible than that of mid-arm circumference. Second, our findings suggest that measurement of both arm circumference and chest circumference is of little additional value in predicting low-birth-weight babies.

We conclude that simple measurements such as chest circumference and also other mentioned anthropometric measure can simply and practicably identify infants with 
LBW when recording birth weight is not feasible. these measurements are easy to learn and can conveniently be introduced in to the existing system of health care to use by paramedical workers to detect neonate who are LBW and at risk. A color coded, measuring tape may be suggested for use by health workers or family members to identify LBW newborns in home setting.

\section{REFERENCES}

[1] The world health report. The newoborn health that went unnoticed, perinatal mortality. A listing of available information. World Health Organization, Geneva, 1996.

[2] Lawn, J.E., Cousens, S. and Zupan, J. (2005) Lancet neonatal survival steering team: 4 million neonatal deaths: when? Where? Why? Lancet, 365, 891-900.

[3] Gogia, S. and Sachdev, H.S. (2010) Home visits by community health workers to prevent neonatal deaths in developing countries: A systematic review. Bulletin of the World Health Organization, 88, 658

[4] Blanc, A.K. and Wardlaw, T. (2005) Monitoring low birth weight: An evaluation of international estimates and an updated estimation procedure. Bulletin of the World Health Organization, 83, 178-185.

[5] United Nations Children's Fund (UNICEF) (2005) The state of the world's children. New York.

[6] Bang, A., Reddy, M.H. and Deshmukh, M.D. (2002) Child mortality in Maharashtra. Economic Political Weekly, 37, 4947-4965.

[7] Darmstadt, G.L., Bhutta, Z.A., Cousens, S., Adam, T., Walker, N. and de Bernis, L. (2005) Lancet neonatal survival steering team: Evidence-based, cost-effective interventions: How many newborn babies can we save? Lancet, 365, 977-998. doi:10.1016/S0140-6736(05)71088-6

[8] Mullany, L.C., Darmstadt, G.L., Coffey, P., Khatry, S.K., LeClerq, S.C. and Tielsch, J.M. (2006) A low cost, colour coded, hand held spring scale accurately categorises birth weight in low resource settings. Archives of Disease in Childhood, 91, 410-413. doi:10.1136/adc.2005.088781

[9] Ramaiya, C., Msamanga, G., Massawe, S., Mpanju, W. and Nywalle, E. (1994) Newborn's arm circumference as screening tool of low birth weight in temeke pistric, peres salaam, Tunzania. Tropical and Geographical Medicine, 46, 318-321.

[10] Naik, D.B., Kulkarni, A.P. and Aswar, N.R. (2003) Birth weight and anthropometry of newborns. Indian Journal of Pediatrics, 2003, 70, 145-146. doi:10.1007/BF02723742

[11] Samal, G.C. and Swain, A.K. (2001) Calf circumference as an alternative to birth weight to predict low birth weight babies. Indian Pediatrics, 38, 275-277.

[12] Gupta, V., Hatwal, S.K., Mathur, S., Tripathi, V.N., Sharma, S.N., Saxena, S.C. and Khadwal, A. (1996) Calf circumference as a predictor of low birth weight babies. Indian Pediatrics, 33, 119-121.

[13] Virdi, U.S., Jain, B.K. and Singh, H. (2001) Calf cir- cumference for identification of low birth weight babies. Indian Pediatrics, 38, 934-935.

[14] World Health Organization (1994) Multi-centre study on low birth weight and infant mortality in India, Nepal and Sri Lanka. Southeast Asia Regional Office, World Health Organization, New Delhi.

[15] Ahmed, F.U., Karim, E. and Bhuiyan, S.N. (2000) Mid-arm circumference at birth as predictor of low birth weight and neonatal mortality. Journal of Biosocial Science, 32, 487-493. doi:10.1017/S0021932000004879

[16] Das, J.C., Afroze, A., Khanam, S.T. and Paul, N. (2005) Mid-arm circumference: an alternative measure for screening low birth weight babies. Bangladesh Medical Research Council Bulletin, 31, 1-6.

[17] Ezeaka, V.C., Egri-Okwaji, M.T., Renner, J.K. and Grange, A.O. (2003) Anthropometric measurements in the detection of low birth weight infants in Lagos. The Nigerian Postgraduate Medical Journal, 10, 168-172.

[18] Hossain, M.M., Habib, M. and DuPont, H.L. (1994) Association between birth weight and birth arm circumference of neonates in rural Egypt. Indian Journal of Pediatrics, 61, 81-87. doi:10.1007/BF02753565

[19] (1993) Use of a simple anthropometric measurement to predict birth weight. WHO collaborative study of birth weight surrogates. Bulletin of the World Health Organization, 71, 157-163.

[20] Mullany, L.C., Darmstadt, G.L., Jhatry, S.K. et. al. (2007) Relationship between the surrogate anthropometric measures, foot length and chest circumference and birth weight among newborn of sarlahi, Nepal. European Journal of Clinical Nutrition, 61, 40-46. doi:10.1038/sj.ejcn.1602504

[21] Hanimi, R.M. and Abhay T. (2005) How to identify neonate at risk of death in rural India: clinical criteria for the risk approach. Journal of Perinatology, 25, s44-s50. doi:10.1038/sj.jp.7211272

[22] Kliegman, R.M., Jenson, H.B., Marcdanle, K.J. and Beharman, R.E. (2006) Nelson essentials of pediatrics. 5th Edition, Elsevier Saunders Press, Amsterdam.

[23] Eghba, L.F. (2007) Evaluation of etiology of low birth weight. Iranian Pediatric Journal, 17, 27-33.

[24] Bindu, N.R., Elizabeth, K.E., Varghese, G. and Varghese, S. (2006) Mid arm circumference (MAC) and body mass index $(\mathrm{BMJ})$ - The tow important auxologic parameters in neonates. Journal of tropical pediatrics, 52, 341-345.

[25] Seeramarddy, C.T., Chuni, N., Patil, R., Singh, D. and Shakya, B. (2008) Anthropometric surrogates to identify low birth weight Nepalese newborn: A hospital-based study. BMC Pediatrics, 8, 16. doi:10.1186/1471-2431-8-16

[26] (1993) WHO Collaborative study of birth weight surrogates-Use of a simple anthropometric measurement to predict birth weight. Bulletin of the World Health Organization, 71, 157-163.

[27] Gupta, V., Hatwal, S.K., Mathur, S., et al. (1996) Calf circumference as a predictor of low birth babies. Indian Pediatrics, 33, 119-121. 